\section{Invasive aspergillosis in an immunocompetent patient with fever and a cardiac mass}

\author{
Matthew LaBarbera, Lester B. Jacobson \\ California Pacific Medical Center, San \\ Francisco, California, CA, USA
}

\begin{abstract}
Invasive aspergillosis is an often fatal disease that usually occurs in immunocompromised patients. We report a case of invasive aspergillosis presenting as a febrile respiratory infection with a cardiac mass in an immunocompetent patient. Invasive asper gillosis should be considered in the differential diagnosis of an otherwise undiagnosed invasive febrile respiratory illness, even in immunocompetent patients. Echocardiography should be performed to evaluate for endocarditis in such cases. Prompt initiation of appropriate antifungal therapy is warranted, even before the diagnosis of invasive aspergillosis is confirmed.
\end{abstract}

\section{Brief Report}

A generally healthy 53 year-old male plumber with suboptimally-controlled noninsulin dependent diabetes mellitus ( $\mathrm{Hb} \mathrm{Alc}$ 8 ) and coronary artery disease s/p CABG 10 years earlier presents to the ED with 10 days of fever and cough. Physical exam reveals a temperature of $36.6^{\circ} \mathrm{C}$, blood pressure of $111 / 70$ $\mathrm{mmHg}$, pulse of $108 \mathrm{bpm}$, respiratory rate of 30 , and pulse oxygen saturation of $80 \%$ on room air which rises to $95 \%$ on 4 liter nasal cannula. There is a regular tachycardia without murmur; rales are present one-quarter up the lung fields bilaterally. Labs are notable for a wbc 15.2 with $73 \%$ neutrophils and $8 \%$ bands. Electrocardiogram demonstrates sinus tachycardia at 110 beats per minute with an incomplete right bundle branch block pattern. Chest radiography reveals bilateral interstitial infiltrates with small nodules in the right upper and left lower lobes. Moxifloxacin is started for treatment of community-acquired pneumonia. 0n HD \#2 CT angiogram of the pulmonary arteries reveals a small left upper lobe pulmonary embolism and extensive bilateral ground-glass opacities. Lower extremity venous Doppler ultrasound study reveals bilateral DVTs below the knee. Therapeutic enoxaparin injections are started. On HD \#3 a bronchoalveolar lavage is performed with washings sent for Gram stain, and bacterial, fungal, AFB, and viral cultures, all of which are negative. Serum human immunodeficiency virus test is negative.

Transthoracic echocardiogram performed on HD \#3 to assess pulmonary artery pressures and cardiac function reveals normal left ventricular systolic function, an estimated right ventricular systolic pressure of $58 \mathrm{mmHg}$, and a mobile $2 \mathrm{~cm} \times 1 \mathrm{~cm}$ mass in the right ventricle adjacent to the tricuspid valve (Figure 1). An infectious disease consultant feels that a fungal etiology for the patient's illness is unlikely as, aside from suboptimally-controlled diabetes mellitus, he has no history of immunocompromise; antibiotics are changed from moxifloxacin to vancomycin and piperacillin-tazobactam. In response to ongoing fevers and progressive hypoxia, a chest CT with contrast is performed on HD \#6, demonstrating a new $5.2 \mathrm{~cm} \times 4.4 \mathrm{~cm}$ cavitating mass in the right upper lobe. Anticoagulation is stopped and fluconazole therapy begun given concern for possible coccidioidomycosis. On HD \#9, a CT-guided transcutaneous biopsy of the right upper lobe mass is performed: Gram stain is negative, as is Gomori methenamine silver stain (GMS) for fungi; fungal cultures are negative. A serum galactomannan test is negative. The patient is started on amphotericin B given his lack of response to broad spectrum antibiotics. He becomes progressively more hypoxic and hypotensive, and is intubated and started on vasopressors.

An endobronchial ultrasound-guided lung and lymph node biopsy is performed on HD \#11. GMS staining from the lung tissue reveals septated fungal structures with branching hyphae characteristic for Aspergillus species. The patient is started on voriconazole. The patient becomes hemodynamically unstable on multiple vasoactive medications, develops multi-organ system failure and dies on HD \#16

On autopsy, examination of the heart confirms a $1.5 \mathrm{~cm} \times 1 \mathrm{~cm}$ RV mass adherent to the tricuspid valve apparatus. GMS staining from lung tissue reveals characteristic fungal forms of Aspergillus (Figure 2). GMS staining of a sample of the right ventricular mass structure reveals a septated fungal form consistent with Aspergillus (Figure 3). Fungal cultures from the lung and heart autopsy specimens show no growth.

\section{Discussion}

Invasive aspergillosis is an often fatal disease, with overall mortality rates of over $50 \%{ }^{1}$ Mortality rates in multisystem or CNS invasive aspergillosis are even higher, and in cases of Aspergillus endocarditis the mortality rate is over $90 \% .^{1-3}$ Invasive aspergillosis typically occurs in immunosuppressed patients, includ-
Correspondence: Lester B. Jacobson, California Pacific Medical Center, 2351 Clay St.

Room 513F, San Francisco, CA 94115, USA. Tel. +1.415.923.3565 - Fax: +1.415.923.3564. E-mail: drlbjake@gmail.com

Key words: invasive aspergillosis, endocarditis, immunocompetent.

Acknowledgements: the authors have no acknowledgments to declare.

Conflict of interest: the authors report no conflicts of interest.

Contributions: MLB, manuscript writing and contents revision; LBJ manuscript editing and patient medical care.

Received for publication: 4 January 2011. Accepted for publication: 1 0ctber 2011.

This work is licensed under a Creative Commons Attribution NonCommercial 3.0 License (CC BYNC 3.0).

(C) Copyright M.LaBarbera and L.B. Jacobson, 2011 Licensee PAGEPress, Italy

Infectious Disease Reports 2011; 3:e12

doi:10.4081/idr.2011.e12

ing those with bone marrow or solid organ transplants, hematologic malignancies, acquired immune deficiency syndrome, or chronic steroid use. ${ }^{1}$ The annual incidence of invasive Aspergillus infection in immunocompromised patients ranges from $0.1-3.9 \%$; only $0.7-6 \%$ of patients with invasive aspergillosis have cardiac involvement. ${ }^{4,5}$ Risk factors for developing fungal (including Aspergillus species) endocarditis include prior cardiac valve surgery, antibiotic use, major non-cardiac surgery, and rheumatic heart disease. ${ }^{2}$ Diabetes mellitus was noted as a risk factor for the development of fungal endocarditis in $4 \%$ of patients in a large case series, while $2 \%$ of patients had no identifiable risk factor. ${ }^{2}$ Establishing a diagnosis of invasive aspergillosis may be difficult, as blood cultures and serologic tests are often negative. ${ }^{3}$

Our case is presented to highlight that invasive aspergillosis may occur in patients with diabetes mellitus and no other risk factors for the development of invasive fungal infections. Second, there was significant delay in starting appropriate treatment partly due to the fastidious nature of Aspergillus in culture media. We speculate that resection of the lung cavity or removal of the cardiac mass early in the patient's course may have expedited diagnosis and allowed earlier treatment. ${ }^{6}$ Lastly, the finding of only a single fungal organism within the tricuspid mass is noteworthy.

Although invasive aspergillosis occurs most 
commonly in immunosuppresed patients, this report highlights a case of invasive aspergillosis with endocarditis in an immunocompetent patient. Patients with Aspergillus endocarditis present with a variety of signs and symptoms, most commonly fever, changing or new heart murmurs, peripheral embolization, focal or generalized neurologic signs, heart failure,

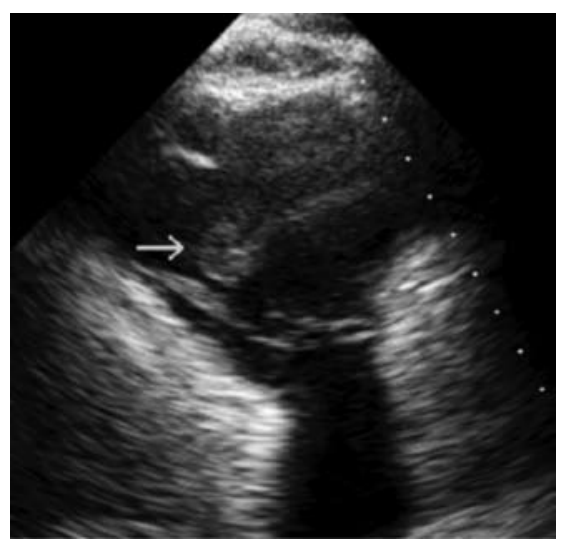

Figure 1. Right ventricular inflow view on echocardiogram showing a $2 \mathrm{~cm} \times 1 \mathrm{~cm}$ right ventricular mass (arrow).

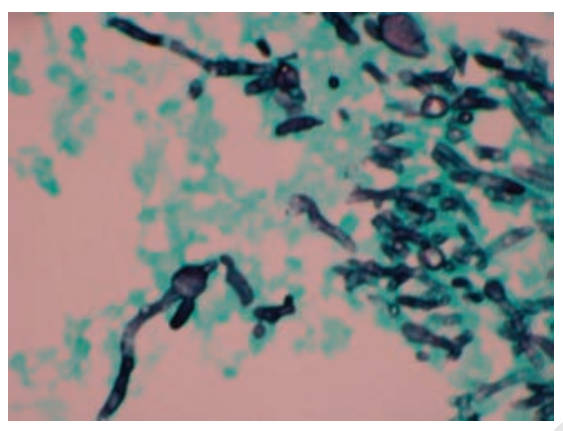

Figure 2. Gomori methenamine silver stain (GMS) of lung tissue from autopsy showing branching hyphae characteristic for Aspergillus (200x). and dyspnea; classic signs of infective endocarditis, such as splinter hemorrhages or Osler's nodes, are rare. ${ }^{2}$ We encourage physicians to entertain the diagnosis of aspergillosis in the setting of an undiagnosed febrile illness involving the respiratory tract, and to use echocardiography in this setting to look for cardiac masses. Infection with Aspergillus should be suspected in patients with progressive culture-negative pneumonia and a cardiac mass. While a serum galactomannan assay may be helpful in establishing a diagnosis, our case highlights the limited sensitivity of this test. ${ }^{7}$ In patients with suspected invasive Aspergillus infection, early attempts to establish a tissue diagnosis should be made and appropriate antifungal therapy should be administered early in the illness. In patients with invasive Aspergillus infection who do not improve with a single antifungal agent, consideration should be given to combination antifungal therapy. ${ }^{8}$ We hope to heighten the awareness of this often fatal disease and urge prompt and aggressive measures to obtain tissue for diagnosis, and perhaps to start appropriate antifungal treatment even before the diagnosis is made.

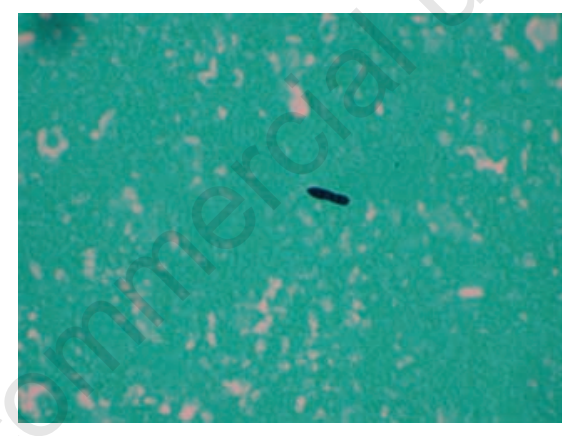

Figure 3. Gomori methenamine silver stain (GMS) of tricuspid valve tissue from autopsy showing a single fungal form $(400 x)$.

\section{References}

1. Lin SJ, Schranz J, Teutsch SM. Aspergillosis case-fatality rate: systematic review of the literature. Clin Infect Dis 2001;32:358-66.

2. Ellis ME, Al-Abdely H, Sandridge A, et al. Fungal endocarditis: evidence in the world literature, 1965-1995. Clin Infect Dis 2001;32:50-62.

3. Gumbo T, Taege AJ, Mawhorter S, et al. Aspergillus valve endocarditis in patients without prior cardiac surgery. Medicine 2000;79:261-8.

4. Morgan J, Wannemuehler KA, Marr KA, Hadley $\mathrm{S}$ et al. Incidence of invasive aspergillosis following hematopoietic stem cell and solid organ transplantation: interim results of a prospective multicenter surveillance program. Med Mycol 2005;43 Suppl1:S49-58.

5. Xie L, Gebre W, Szabo K, Lin J. Cardiac aspergillosis in patients with acquired immunodeficiency syndrome: a case report and review of the literature. Arch Pathol Lab Med 2005;129:511-5.

6. Ryu KM, Seo PW, Kim SH, et al. Surgical treatment of native valve aspergillus endocarditis and fungemic vascular complications. J Korean Med Sci 2009;24:170-2.

7. Pfeiffer CD, Fine JP, and Safdar N. Diagnosis of invasive aspergillosis using a galactomannan assay: a meta-analysis. Clin Infect Dis 2006;42:1417-27.

8. Steinbach WJ, Stevens DA, Denning DW. Combination and sequential antifungal therapy for invasive aspergillosis: review of published in vitro and in vivo interactions and 6281 clinical cases from 1966 to 2001. Clin Infect Dis 2003;37Suppl3:S188224. 